\title{
An improved multivariable integrated evaluation method and tool (MVIETool) v1.0 for multimodel intercomparison
}

\author{
Meng-Zhuo Zhang ${ }^{1,2}$, Zhongfeng $\mathrm{Xu}^{2,3}$, Ying $\mathrm{Han}^{2}$, and Weidong Guo ${ }^{1,3}$ \\ ${ }^{1}$ Institute for Climate and Global Change Research, School of Atmospheric Sciences, Nanjing University, Nanjing, China \\ ${ }^{2}$ CAS Key Laboratory of Regional Climate and Environment for Temperate East Asia, Institute of Atmospheric Physics, \\ Chinese Academy of Sciences, Beijing, China \\ ${ }^{3}$ Jiangsu Collaborative Innovation Center for Climate Change, Nanjing University, Nanjing, China
}

Correspondence: Zhongfeng Xu (xuzhf@tea.ac.cn)

Received: 16 September 2020 - Discussion started: 27 November 2020

Revised: 8 April 2021 - Accepted: 23 April 2021 - Published: 28 May 2021

\begin{abstract}
An evaluation of a model's overall performance in simulating multiple fields is fundamental to model intercomparison and development. A multivariable integrated evaluation (MVIE) method was proposed previously based on a vector field evaluation (VFE) diagram, which can provide quantitative and comprehensive evaluation on multiple fields. In this study, we make further improvements to this method from the following aspects. (1) We take area weighting into account in the definition of statistics in the VFE diagram and MVIE method, which is particularly important for a global evaluation. (2) We consider the combination of multiple scalar fields and vector fields against multiple scalar fields alone in the previous MVIE method. (3) A multivariable integrated skill score (MISS) is proposed as a flexible index to measure a model's ability to simulate multiple fields. Compared with the multivariable integrated evaluation index (MIEI) proposed in the previous study, MISS is a normalized index that can adjust the relative importance of different aspects of model performance. (4) A simple-to-use and straightforward tool, the Multivariable Integrated Evaluation Tool (MVIETool version 1.0), is developed to facilitate an intercomparison of the performance of various models. Users can use the tool coded either with the open-source NCAR Command Language (NCL) or Python3 to calculate the MVIE statistics and plotting. With the support of this tool, one can easily evaluate model performance in terms of each individual variable and/or multiple variables.
\end{abstract}

\section{Introduction}

An increasing number of model intercomparison projects (MIPs) have been carried out over the past decade (Eyring et al., 2016; Simpkins, 2017). The Coupled Model Intercomparison Project phase 6 (CMIP6) includes more than 20 MIPs: e.g., the Radiative Forcing MIP (RFMIP), the Geoengineering MIP (GeoMIP), and the Global Monsoons MIP (GMMIP) (Kravitz et al., 2011; Pincus et al., 2016; Zhou et al., 2016). Quantitative evaluation and intercomparison of climate models have become increasingly important (Knutti et al., 2013) and have escalated the need for innovative and comprehensive approaches to model evaluation (Meehl et al., 2014; Stouffer et al., 2016; Eyring et al., 2019).

Climate models are commonly evaluated in terms of their ability to simulate historical climate compared to observed or reanalyzed data, using performance metrics (Pincus et al., 2008; Flato et al., 2013). A set of useful metrics and diagrams has been developed for model evaluation. The widely used Taylor diagram summarizes model performance in simulating a scalar field using correlation coefficient (CORR), standard deviation (SD), and root-mean-square difference (Taylor, 2001). Objective performance metrics (e.g., relative error and portrait diagrams) have been proposed for the evaluation of various variables (Gleckler et al., 2008). Xu et al. (2016) devised a vector field evaluation (VFE) diagram which can be regarded as a generalized Taylor diagram. The VFE method allows an evaluation of a model's ability to simulate a vector field (Huang et al., 2019, 2020). Based on the VFE diagram, Xu et al. (2017) further developed a multivariable in- 
tegrated evaluation (MVIE) method to evaluate model performance in terms of multiple fields by grouping various normalized scalar fields into an integrated vector field. The MVIE method also defined a multivariable integrated evaluation index (MIEI) to summarize the model's overall performance in simulating multiple fields. The MIEI, the VFE diagram, and the performance metrics of individual scalar variables constitute a hierarchical model evaluation framework, which can provide a quantitative and comprehensive evaluation of model performance.

However, the MVIE method proposed by Xu et al. (2017) considered only the integrated evaluation of various scalar fields. Under certain circumstances, both scalar variables and vector variables (e.g., air temperature and vector wind fields) warrant evaluation together. Moreover, the vector field statistics employed in Xu et al. $(2016,2017)$ did not consider area weighting, which is a limitation especially for an evaluation of the global field. Although area weighting was considered in many previous statistical metrics, e.g., correlation coefficient and standard deviation, they were used to evaluate scalar fields rather than vector fields (e.g., Watterson, 1996; Boer and Lambert, 2001; Masson and Knutti, 2011). The consideration of area weighting in the definition of vector field statistics is one of the novelty of our study relative to previous studies (Taylor, 2001; Boer and Lambert, 2001; Gleckler et al., 2008; Xu et al., 2016, 2017). In addition, we also improve the MVIE method to allow a mixed evaluation of scalar and vector fields. Furthermore, based on MIEI, a multivariable integrated skill score (MISS) for a climate model is proposed, which allows us to adjust the relative importance of different aspects of model performance. Finally, we develop a Multivariable Integrated Evaluation Tool (MVIETool version 1.0) to facilitate multimodel intercomparison. These efforts are expected to improve the accuracy and flexibility of the VFE and MVIE methods.

The paper is organized as follows. Section 2 defines statistical metrics that take area weighting into account. Section 3 introduces the improved MVIE method with a combination of scalar and vector fields, and interprets the performance metrics. Section 4 gives an overview of the MVIETool and describes the technical process of the MVIETool, including the setting of the arguments in scripts. In Sect. 5, the applications of the tool are demonstrated by showing three examples with 10 CMIP phase 5 (CMIP5) models. Finally, a summary is given in Sect. 6.

\section{Statistical metrics}

The MVIE method primarily consists of three statistical quantities - root-mean-square length (RMSL), vector similarity coefficient (VSC), and root-mean-square vector difference (RMSVD) - that measure model performance in simulating a vector field from various aspects (Xu et al., 2017). RMSL measures the magnitude of a vector field, VSC mea- sures the similarity of two vector fields, and RMSVD measures the overall difference between two vector fields. MIEI was defined by using root-mean-square (rms) values of all variables and VSC, and is a concise metric to rank models in terms of their performance in simulating multiple fields. However, the definition of these statistical quantities did not consider area weighting, which could to a certain extent misrepresent the relative contribution of different latitudes to the statistics. Here, we redefine these statistical quantities by taking area weighting into account.

Assume that there are $M$ variables derived from model $A$ and observation $O$. We need to normalize each modeled variable using the rms value of the corresponding observed variable. The normalized $M$ variables are dimensionless and can be grouped into $M$-dimensional vector fields for model $\boldsymbol{A}$ and observation $\boldsymbol{O}$ :

$$
\begin{aligned}
& \boldsymbol{A}_{j}=\left(a_{1 j}, a_{2 j}, \ldots, a_{M j}\right) ; j=1,2, \ldots, N \\
& \boldsymbol{O}_{j}=\left(o_{1 j}, o_{2 j}, \ldots, o_{M j}\right) ; j=1,2, \ldots, N .
\end{aligned}
$$

Each field is composed of $N$ vectors in time and/or space and $M$ is the dimension of the integrated vector field.

\subsection{Uncentered statistics}

Similar to the weighted statistics defined by Watterson (1996), we define the weighted RMSL $\left(L_{A}, L_{O}\right)$, VSC $\left(R_{\mathrm{V}}\right)$, and RMSVD as follows:

$$
\begin{aligned}
& L_{A}=\sqrt{\sum_{i=1}^{M} \sum_{j=1}^{N} w_{j} a_{i j}^{2},} \\
& L_{O}=\sqrt{\sum_{i=1}^{M} \sum_{j=1}^{N} w_{j} o_{i j}^{2}} \\
& R_{\mathrm{V}}=\frac{\sum_{i=1}^{M} \sum_{j=1}^{N} w_{j} a_{i j} o_{i j}}{L_{A} \cdot L_{O}} \\
& \text { RMSVD }=\sqrt{\sum_{i=1}^{M} \sum_{j=1}^{N} w_{j}\left(a_{i j}-o_{i j}\right)^{2}},
\end{aligned}
$$

where $w_{j}$ is the area weighting factor and the sum of $w_{j}$ is equal to 1 . In terms of equal weight, $w_{j}$ is equal to $1 / N$ for all $j$. These uncentered metrics focus mainly on different aspects of the vector field. With the aid of Eq. (3), the square 
of RMSVD can be written as

$$
\begin{aligned}
\operatorname{RMSVD}^{2}= & \sum_{i=1}^{M} \sum_{j=1}^{N} w_{j}\left(a_{i j}-o_{i j}\right)^{2} \\
= & \sum_{i=1}^{M} \sum_{j=1}^{N} w_{j}\left(a_{i j}^{2}+o_{i j}^{2}-2 \cdot a_{i j} \cdot o_{i j}\right) \\
= & \sum_{i=1}^{M} \sum_{j=1}^{N} w_{j} a_{i j}^{2} \\
& +\sum_{i=1}^{M} \sum_{j=1}^{N} w_{j} o_{i j}^{2}-2 \cdot \sum_{i=1}^{M} \sum_{j=1}^{N} w_{j} a_{i j} o_{i j} .
\end{aligned}
$$

With the aid of Eqs. (1)-(3), Eq. (4) can be written as

$\operatorname{RMSVD}^{2}=L_{A}^{2}+L_{O}^{2}-2 \cdot L_{A} \cdot L_{O} \cdot R_{\mathrm{V}}$

Note that RMSVD, $L_{A}, L_{O}$, and $R_{\mathrm{v}}$ with area weighting still satisfy the cosine law (Eq. 5). Thus, the VFE diagram is still valid with these weighted statistics (Eq. 5). We define the standard deviation of rms values (rms_std) to quantify the dispersion of the rms values of $M$ variables:

$\sigma_{\mathrm{rms}}=\sqrt{\frac{1}{M} \sum_{m=1}^{M}\left(L_{A_{m}}^{*}-\frac{1}{M} \sum_{m=1}^{M} L_{A_{m}}^{*}\right)^{2}}$,

where $L_{A_{m}}^{*}=\frac{L_{A_{m}}}{L_{O_{m}}}$ is the ratio of the modeled rms value of the $m$ th component (variable) to the observed rms value.

Note that RMSVD does not decrease monotonically with an improvement in model performance. To measure model performance more accurately, Xu et al. (2017) devised a multivariable integrated evaluation index, termed MIEI, of climate model performance:

$\mathrm{MIEI}^{2}=\frac{1}{M} \sum_{m=1}^{M}\left(L_{A_{m}}^{*}-1\right)^{2}+2 \cdot\left(1-R_{\mathrm{V}}\right)$.

Note that the first and second terms on the right-hand side of Eq. (7a) can vary from 0 to $+\infty$ and from 0 to 4 , respectively. Thus, the MIEI may be too sensitive to rms bias and insensitive to pattern bias. To fix this problem, we redefine MIEI as follows:

$\mathrm{MIEI}^{2}=\frac{1}{M} \sum_{m=1}^{M}\left(R_{m}^{*}-1\right)^{2}+2 \cdot\left(1-R_{\mathrm{V}}\right)$,

where $R_{m}^{*}$ is defined as

$R_{m}^{*}=\left\{\begin{array}{cc}L_{A_{m}}^{*}, & L_{A_{m}}^{*} \leq 1 \\ \frac{1}{L_{A_{m}}^{*}}, & L_{A_{m}}^{*}>1\end{array}\right.$

$R_{m}^{*}$ varies from 0 to 1 . Here, we assume that $L_{A_{m}}^{*}$ and $\frac{1}{L_{A_{m}}^{*}}$ represent the same model performance except that one overestimates rms and the other underestimates rms. MIEI takes the rms values and VSC into consideration at the same time.
The relative importance of a model's ability to simulate the pattern similarity and amplitude of variables depends on the application. Hence, a weight factor $F$ is added to the MIEI to adjust the relative importance of rms and VSC:

$\mathrm{MIEI}^{2}=\frac{1}{M} \sum_{m=1}^{M}\left(R_{m}^{*}-1\right)^{2}+F \cdot\left(1-R_{\mathrm{v}}\right)$.

We can further define a multivariable integrated skill score (MISS) of a climate model:

$\operatorname{MISS}=\left(F+1-\mathrm{MIEI}^{2}\right) /(F+1)$.

MISS varies from $-F /(F+1)$ to 1 . MISS reaches its minimum value of $-F /(F+1)$ when $R_{\mathrm{v}}$ equals -1 and $R_{m}^{*}$ equals 0 . Note that $R_{\mathrm{V}}$ is usually greater than 0 in terms of model evaluation. Thus, we find that MISS usually varies from 0 to 1 . It is very unlikely that MISS will be less than 0 , unless $R_{\mathrm{v}}$ is less than 0 . MISS varies monotonically with respect to model performance and reaches its maximum value of 1 when the model performs best. With an increase in $F$, MISS is less (more) sensitive to the model's ability to simulate amplitudes (patterns).

In terms of climate model evaluation, the pattern similarity is usually more important than the amplitude, because without pattern similarity, the accuracy of amplitude simulation is often less meaningful. Thus, one can set $F$ to be a value greater than 1 in Eq. (10) for a general model evaluation purpose. In this case, MISS/MIEI is more sensitive to the change in the pattern similarity than the amplitude. Considering that MIEI has a geometric meaning when $F$ is 2, which represents the length of a line segment (referring to the hypotenuse of a right triangle from point $\mathrm{C}$ to $\mathrm{G}$ in Fig. 3 in $\mathrm{Xu}$ et al., 2017). Thus, 2 appears to be a reasonable value of $F$ for general model evaluation purpose. Users can also change $F$ based on the application. For example, one may use a smaller $F$, say $F=0.5$, to give more weight to the amplitude if one wants to evaluate model ability to simulate the long-term trend of the multiple variables, e.g., the surface air temperature and specific humidity. In this case, one may have more concern about the values of the trends than their spatial patterns.

\subsection{Centered statistics}

As well as uncentered statistics, centered statistics are also important when the anomaly field is the main concern of model evaluation. For the centered mode, centered RMSL (cRMSL), centered VSC (cVSC), and centered RMSVD (cRMSVD) with area weighting are defined to evaluate the model performance in terms of anomaly fields. The centered statistics are the same as the uncentered statistics, except that the original field is replaced by the anomaly field. These 
statistics are written as follows:

$$
\begin{aligned}
& \mathrm{c} L_{A}=\sqrt{\sum_{i=1}^{M} \sum_{j=1}^{N} w_{j}\left(a_{i j}-\bar{a}_{i}\right)^{2}}, \\
& \mathrm{c} L_{O}=\sqrt{\sum_{i=1}^{M} \sum_{j=1}^{N} w_{j}\left(o_{i j}-\bar{o}_{i}\right)^{2}} \\
& \mathrm{c} R_{\mathrm{V}}=\frac{\sum_{i=1}^{M} \sum_{j=1}^{N} w_{j}\left(a_{i j}-\bar{a}_{i}\right)\left(o_{i j}-\bar{o}_{i}\right)}{\mathrm{c} L_{A} \cdot \mathrm{c} L_{o}} \\
& \mathrm{cRMSVD}=\sqrt{\sum_{i=1}^{M} \sum_{j=1}^{N} w_{j}\left[\left(a_{i j}-\bar{a}_{i}\right)-\left(o_{i j}-\bar{o}_{i}\right)\right]^{2} .}
\end{aligned}
$$

One can use the vector mean error (VME) to additionally measure the difference between two mean vector fields since the mean difference was removed from the centered statistics mentioned above. The VME can also be written as the rootmean-square error of two mean fields:

$$
\begin{aligned}
& \mathrm{VME}=\sqrt{\sum_{i=1}^{M}\left(\bar{a}_{i}-\bar{o}_{i}\right)^{2},} \\
& \bar{a}_{i}=\sum_{j=1}^{N} w_{j} a_{i j}, \\
& \bar{o}_{i}=\sum_{j=1}^{N} w_{j} o_{i j} .
\end{aligned}
$$

As the uncentered statistics (Eqs. 1-3) can be transformed into centered statistics (Eqs. 11-13), by replacing the original field with the anomaly field, cRMSL, cVSC, and cRMSVD also satisfy the cosine law:

$\mathrm{cRMSVD}^{2}=\mathrm{c} L_{A}^{2}+\mathrm{c} L_{O}^{2}-2 \cdot \mathrm{c} L_{A} \cdot \mathrm{c} L_{O} \cdot \mathrm{c} R_{\mathrm{v}}$.
Furthermore, with the aid of Eq. (3), we can decompose the square of RMSVD as follows:

$$
\begin{aligned}
\operatorname{RMSVD}^{2}= & \sum_{i=1}^{M} \sum_{j=1}^{N} w_{j}\left\{\left[\left(a_{i j}-\bar{a}_{i}\right)-\left(o_{i j}-\bar{o}_{i}\right)\right]\right. \\
& \left.+\left(\bar{a}_{i}-\bar{o}_{i}\right)\right\}^{2} \\
= & \sum_{i=1}^{M} \sum_{j=1}^{N} w_{j}\left[\left(a_{i j}-\bar{a}_{i}\right)-\left(o_{i j}-\bar{o}_{i}\right)\right]^{2} \\
& +\sum_{i=1}^{M} \sum_{j=1}^{N} w_{j}\left(\bar{a}_{i}-\bar{o}_{i}\right)^{2} \\
& +2 \cdot \sum_{i=1}^{M}\left\{\left(\bar{a}_{i}-\bar{o}_{i}\right)\right. \\
& \left.\sum_{j=1}^{N} w_{j}\left[\left(a_{i j}-\bar{a}_{i}\right)-\left(o_{i j}-\bar{o}_{i}\right)\right]\right\} .
\end{aligned}
$$

With the aid of Eqs. (13)-(14), RMSVD, cRMSVD, and VME satisfy the Pythagorean theorem:

$$
\begin{aligned}
\mathrm{RMSVD}^{2} & =\mathrm{VME}^{2}+\mathrm{cRMSVD}^{2} \\
& =\mathrm{VME}^{2}+\mathrm{c} L_{A}^{2}+\mathrm{c} L_{O}^{2}-2 \cdot \mathrm{c} L_{A} \cdot \mathrm{c} L_{O} \cdot \mathrm{c} R_{\mathrm{v}} .
\end{aligned}
$$

Clearly, these statistics for vector variable evaluation satisfy the cosine law and Pythagorean theorem. Similarly, such relationships are also valid for scalar variables (Taylor, 2001; $\mathrm{Xu}$ and Han, 2019).

Similar to rms_std (Eq. 6), the standard deviation of SD (SD_std) is also defined to describe the dispersion of SD over all variables:

$\sigma_{\mathrm{SD}}=\sqrt{\frac{1}{M} \sum_{m=1}^{M}\left(\mathrm{c} L_{A_{m}}^{*}-\frac{1}{M} \sum_{m=1}^{M} \mathrm{c} L_{A_{m}}^{*}\right)^{2}}$,

where $\mathrm{c} L_{A_{m}}^{*}$ is the same as $L_{A_{m}}^{*}$, except that it is the ratio of SDs.

\section{MVIE with a combination of multiple scalar and vector fields}

The MVIE method proposed by Xu et al. (2017) considers only multiple scalar fields. Under some circumstances, one may want to simultaneously evaluate both scalar and vector fields. Here, the MVIE method is improved to meet this need. Assume there are $M$ individual variables to be evaluated, which are either scalar or vector fields (the upper left part of Fig. 1). Variable $d_{m}$ is the dimension of the $m$ th variable, where $d_{m}$ is equal to 1 for a scalar field (e.g., temperature), while $d_{m}$ is 2 for a two-dimensional vector field (e.g., a vector 


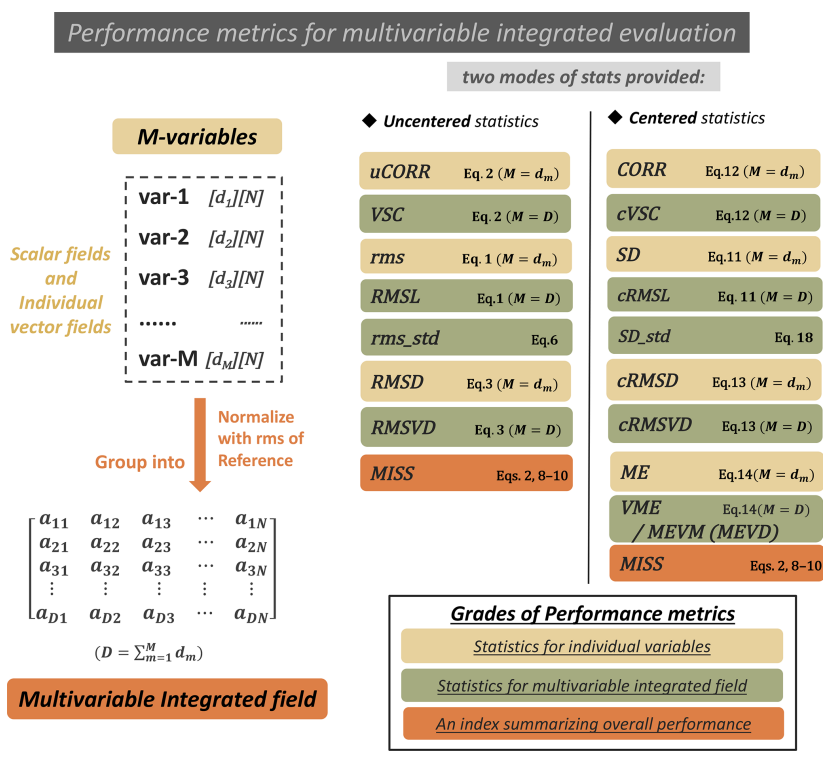

Figure 1. General idea and performance metrics of the improved MVIE. The left-hand column illustrates the general idea of the MVIE. Two modes of statistics are provided for evaluation: uncentered statistics (middle column) and centered statistics (right-hand column). In each mode, the statistics are sorted into three grades: statistics for individual variables, statistics for the multiple field, and a summary index. The right-hand side of the statistics box is its formula.

wind) and so on. Hereafter, the vector field for an individual variable (e.g., a $850 \mathrm{hPa}$ wind field) is termed the individual vector field to separate it from vector fields grouped from multiple fields. Following the idea of MVIE, these variables are normalized with respective rms values of the reference. Note that an individual scalar field is normalized by dividing by its rms value. An individual vector field is normalized as a whole by dividing by its RMSL. These normalized scalar and/or vector fields can be grouped into a multivariable field with the dimension $D \times N$, where $D$ is the sum of $d_{m}$. The multivariable field derived from the model can be evaluated against that derived from observation by using the various performance metrics in the uncentered or centered mode (Fig. 1).

The uncentered mode focuses on the whole original field, while the centered mode separately evaluates the anomaly field and the mean field. Each mode of statistics consists of three levels of statistics: statistics for individual variables (yellow boxes), multivariable integrated statistics (green boxes), and an index summarizing the overall model performance (orange boxes). The definitions of centered and uncentered statistics are the same as those defined by $\mathrm{Xu}$ et al. (2017), except that the area weighting is considered here. To calculate the statistics for individual variables, e.g., root-mean-square difference (RMSD), centered RMSD (cRMSD), or uncentered CORR (uCORR), we can also use the formulas of multivariable integrated statistics (Eqs. 1-3,
11-14) by setting $M$ equal to $d_{m}$, which is summarized in the right-hand part of the boxes in Fig. 1.

Note that the mean error (ME) is additionally computed for the centered statistics, as the centered statistics exclude mean error. For a scalar variable, ME is calculated with Eq. (14) by setting $M$ to 1 , but it is signed. Because VME is a function of the difference in the vector magnitude and direction, we provide two additional statistical metrics - the mean error of vector magnitude (MEVM) and the mean error of vector direction (MEVD) - to separate the magnitude error from the directional error. MEVM (MEVD) is the mean of the magnitude error (direction difference) between the modeled vector and the observed vector for all grids evaluated. Note that the MEVD is only valid for 2-D vector fields. The direction difference ranges from -180 to 180 , and the positive (negative) value represents a counterclockwise (clockwise) directional error of the model mean vector.

To summarize the overall model skill score in terms of the simulation of multiple variables, the uncentered MISS (uMISS) and centered MISS (cMISS) are provided for the uncentered and centered modes, respectively. uMISS is calculated with Eqs. (9) and (10) using the original fields. cMISS can also be calculated with Eqs. (9) and (10) but by replacing the rms and VSC with SD and cVSC, respectively. With the support of these statistics, the improved MVIE method can provide a more comprehensive and precise evaluation of model performance. All statistics defined in this paper together with their acronyms are summarized in Table A1 in the Appendix.

\section{The Multivariable Integrated Evaluation Tool}

\subsection{Brief overview}

The MVIETool consists of two main scripts and some function scripts. All these scripts are written in NCAR Command Language (NCL), which can be easily used in Linux and Mac operating systems. The two main scripts are Calculate_MVIE.ncl and Plot_MVIE.ncl. The execution of the MVIETool can be simplified to two runs, which work independently but in sequence (Fig. 2). Users can modify arguments written in a module at the beginning of the main scripts according to the application. The script assumes that the model data and observation data are saved in Network Common Data Form (NetCDF) format. The Calculate_MVIE.ncl script calculates the statistical metrics defined in this paper. The output of this script is saved in a NetCDF file, which is used as the input to Plot_MVIE.ncl for plotting the VFE diagram and the metrics table.

\subsection{Preparing the input data}

The MVIETool requires two groups of datasets as inputs the model data and observations - saved in NetCDF format. Each model or observational data file includes all the 


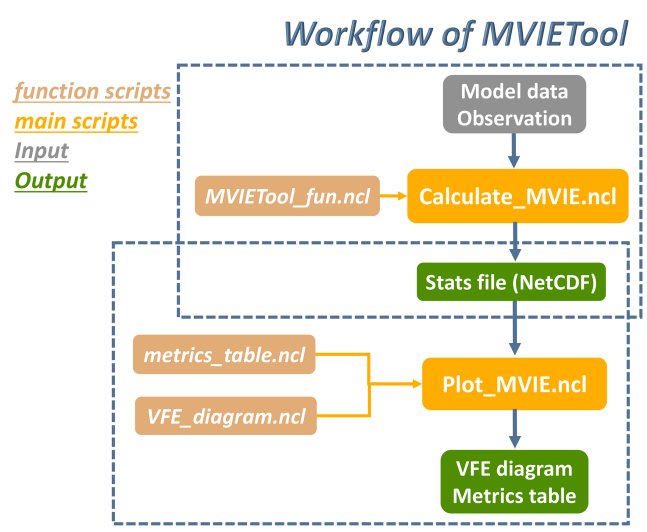

Figure 2. The structure of the MVIETool. The primary input of the workflow is model data and observation (gray) with fixed formats. Inside the dotted blue boxes are two independent runs for calculation and plotting, respectively. Two main scripts (yellow) need to be invoked in sequence. The outputs (green) are the NetCDF file storing performance metrics, the VFE diagram, and the metrics table.

variables to be evaluated. If the variables are saved separately as CMIP data, one can easily merge these variables into one data file by using third-party software: e.g., the Climate Data Operators (CDO) or NetCDF operators (NCO). The main script also assumes that all model and observation data files are stored in the same directory. Therefore, users need to move or link various data into the same directory. Variables stored in the data file need to be on the same grid. Examples are given in the user guide of the MVIETool package to show how to regrid data on regular or irregular grids into the same regular grid with NCL, CDO, and Python3, respectively. In terms of vector variables, each component of the vector variable should be stored independently. If users want to consider area weighting in the statistics, the variables should be saved with the dimension names and the coordinate information (e.g., time, latitude, and longitude), because the coordinate information is needed for the calculation of area weighting. Currently, the tool can only deal with area weighting for regular grids and area weighting is calculated by the formula as

$w_{j}=\sin \left(\mathrm{lat}_{j}+d_{\mathrm{lat}}\right)-\sin \left(\mathrm{lat}_{j}-d_{\mathrm{lat}}\right)$,

where lat ${ }_{j}$ is the latitude in $j$ th grid and $d_{\text {lat }}$ is the difference in latitude between two adjacent zonal grids. The tool can only identify the time and geographical coordinates of regular grid: i.e., time, latitude, longitude, and level.

Figure 3 illustrates an example that consists of 10 models (M1-M10) to be evaluated and two sets of reanalysis (REA1, REA2) data as reference in the same directory. Each data file includes eight variables: $600 \mathrm{hPa}$ specific humidity (Q600), sea level pressure (SLP), sea surface temperature (SST), $850 \mathrm{hPa}$ air temperature (T850), u850, v850, u200, and v200. Among these, u850 (u200) and v850 (v200) are the zonal and meridional components of vector winds in $850 \mathrm{hPa}$

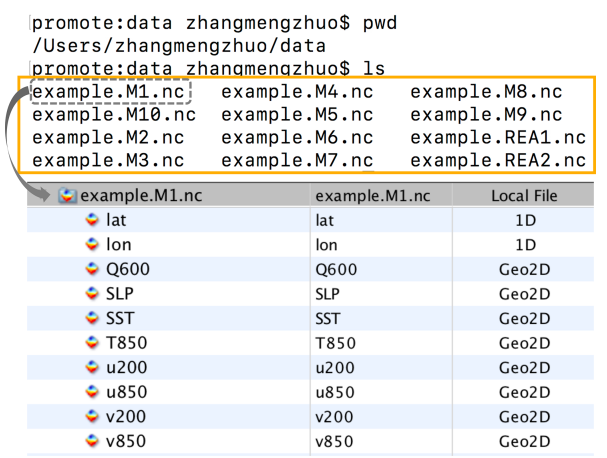

Figure 3. Example of data preparation for the MVIETool. There are eight variables stored in each data file: Q600, SLP, SST, T850, u200, u850, v200, v850. Variables u850 (u200) and v850 (v200) compose an individual 2-D vector variable - uv850 (uv200), and the other four variables are regarded as scalar variables. REA1 and REA2 are two sets of reanalysis data used in the evaluation.

$(200 \mathrm{hPa})$, respectively. The MVIETool allows treating u850 (u200) and v850 (v200) as an individual vector field rather than two scalar fields. To declare a vector field, users can simply put the components of a vector in parenthesis separated by comma, e.g., (u850, v850) and (u200, v200) in the argument Varname of the tool (Table 1). Thus, the evaluation actually includes six individual variables. One can also save various surface variables (e.g., SST) and multi-level variables (e.g., air temperature) in the same file. The MVIETool can only evaluate part of the multi-level variables specified by user.

\subsection{Usage and workflow of the MVIETool}

Once datasets have been prepared, one can use the MVIETool to evaluate model performance. Users should set some arguments at the beginning of Calculate_MVIE.ncl based on the application. The arguments are summarized in Table 1 and discussed in this section. The rightmost column of Table 1 gives an example of argument setting. Arguments 1-5 define the data file information as in the example of Fig. 3. Note that, in the argument Varname in Table 1, the vector variable is identified by enclosing its components in parentheses: e.g., (u200, v200). Notably, if users want to add area weighting to the statistics, the data should have the latitude coordinate, by setting arguments $9-11$. Some arguments are mandatory, e.g., arguments 1-5 in Table 1, while some arguments are optional. We provide a default value for most of the arguments and the default value will be used if users do not specify the argument, except for Range_time, Coords_geo, Range_geo, and VarLev. These four arguments must be set when input variables have corresponding coordinates.

After reading the data, the reference data are calculated with observation and/or reanalysis data. As shown in Fig. 4, if users provide only one observational dataset, it is directly used as the reference data. Otherwise, the mean of multiple 
Table 1. Input arguments to Calculate_MVIE.ncl in the MVIETool. An argument with * is optional. The rightmost column provides examples of these arguments.

\begin{tabular}{|c|c|c|c|}
\hline & Argument & Description & Example \\
\hline 1 & Varname & $\begin{array}{l}\text { Names of independent variables stored in data file. For individual } \\
\text { vector variables, names of its components need to be enclosed in } \\
\text { parentheses separated by a comma. }\end{array}$ & $\begin{array}{l}\text { "Q600, SLP, SST, T850, (u200, v200), } \\
\text { (u850, v850)" }\end{array}$ \\
\hline 2 & Model_filenames & Names of model data files for evaluation. & 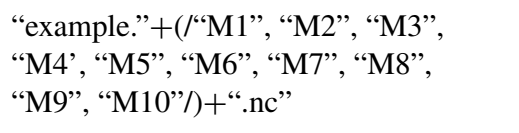 \\
\hline 3 & Obs_filenames & Names of observation and/or reanalysis data files for evaluation. & "example."+(/“REA1","REA2”/)+“.nc” \\
\hline 4 & Inputdatadir & Model and observation data input directory. & “/Users/zhangmengzhuo/data/" \\
\hline 5 & Var_Coords & Whether to read data by the range of coordinates. & True \\
\hline 6 & $*_{\text {isCoords_time }}$ & $\begin{array}{l}\text { Whether evaluated variables have time dimension and coordinate } \\
\text { under Var_Coords is True. }\end{array}$ & True \\
\hline 7 & *Coords_time & $\begin{array}{l}\text { Name of time dimension for evaluated variables under isCo- } \\
\text { ords_time is True. }\end{array}$ & "time" \\
\hline 8 & *Range_time & $\begin{array}{l}\text { Range of time in time coordinate of evaluated variables under isCo- } \\
\text { ords_time is True. }\end{array}$ & (/“19710101”, “19991201”/) \\
\hline 9 & *isCoords_geo & $\begin{array}{l}\text { Whether evaluated variables have latitude and/or longitude dimen- } \\
\text { sions and coordinates under Var_Coords is True. }\end{array}$ & True \\
\hline 10 & *Coords_geo & $\begin{array}{l}\text { Names of latitude and/or longitude dimensions for evaluated vari- } \\
\text { ables under isCoords_geo is True. }\end{array}$ & (/"lat", "lon"/) \\
\hline 11 & *Range_geo & $\begin{array}{l}\text { Ranges of latitude and/or longitude in their coordinates of eval- } \\
\text { uated variables under isCoords_geo is True. It has the format of } \\
\text { (/“lat|0:45", "lon|0:180"/). }\end{array}$ & (/“lat|0:45", "lon|0:180”/) \\
\hline 12 & ${ }^{*}$ hasLevel & $\begin{array}{l}\text { Whether evaluated variables have level dimension. If so, set it to the } \\
\text { level dimension name; otherwise, set False. The level coordinate is } \\
\text { required to read data at specific level. }\end{array}$ & False \\
\hline 13 & *VarLev & $\begin{array}{l}\text { Specify the level for each evaluated variable. If a variable does not } \\
\text { have the level dimension, users can provide an arbitrary value in the } \\
\text { corresponding position to match the variable in Varname. }\end{array}$ & - \\
\hline 14 & *Isarea_wgt & $\begin{array}{l}\text { If no area weighting in statistics, set False; otherwise, set to the } \\
\text { name of the latitude dimension in Coords_geo. }\end{array}$ & "lat" \\
\hline 15 & *Type_stats & Data type of calculated statistics. & "float" \\
\hline 16 & *Stats_mode & $\begin{array}{l}\text { Calculate uncentered/centered statistics, and it is } 1 \text { or } 0 \text {, represent- } \\
\text { ing the uncentered or the centered mode. }\end{array}$ & 0 \\
\hline 17 & *Wgt_var & $\begin{array}{l}\text { Add weights of variables. If not adding, set to } 1 \text {; otherwise, set 1-D } \\
\text { numeric array that should match variables in Varname. }\end{array}$ & $(/ 1,1,2,3,1,1,2,2 /)$ \\
\hline 18 & *ComMask_On & $\begin{array}{l}\text { Create a common mask for all model data and reference (True) or } \\
\text { unify the missing points between each model-reference pair (False). }\end{array}$ & True \\
\hline 19 & *Unif_VarMiss & Whether to unify missing points across all variables of one model. & True \\
\hline 20 & *Cal_VME & $\begin{array}{l}\text { Calculate VME/MEVM in centered mode. Set it to } 1(0) \text {, represent- } \\
\text { ing calculating VME (MEVM). }\end{array}$ & 1 \\
\hline 21 & *MISS_F & Parameter $F$ in the calculation of MISS. & 2 \\
\hline 22 & ${ }^{*}$ Print_stats_r & Whether to print range of performance metrics on the screen. & True \\
\hline 23 & *MVIE_filename & Output NetCDF file name. & "example.centered.nc" \\
\hline
\end{tabular}




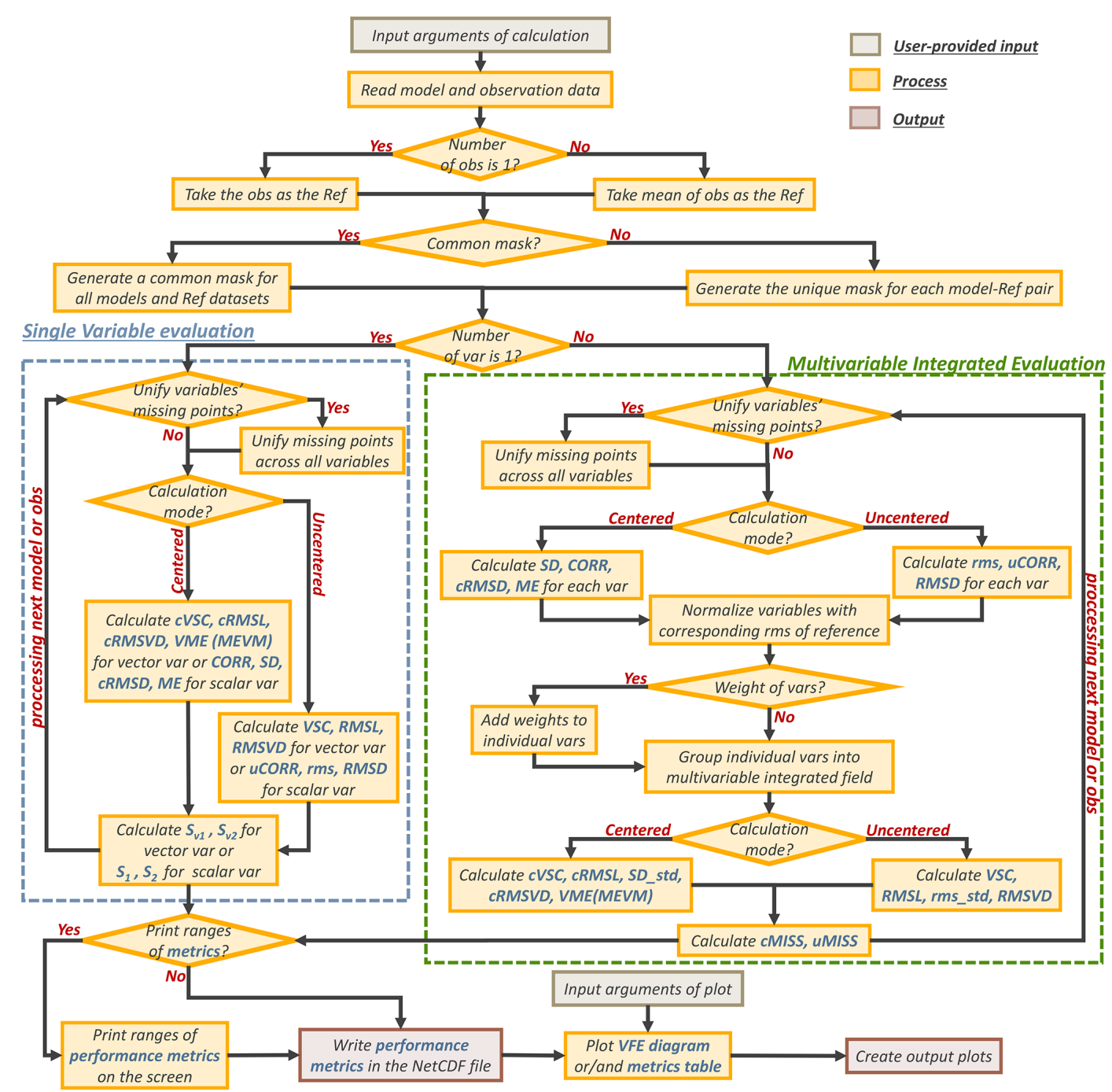

Figure 4. Flow chart of the MVIETool procedure. The dotted blue and green rectangles outline the procedures of single variable evaluation and multivariable integrated evaluation, respectively.

observational datasets is used as the reference and each observational piece of data will also be evaluated against the ensemble mean to measure the observational uncertainty. Considering that some variables may contain missing values and some may not, to make the evaluation comparable between different models, a common mask for all models and the reference data is generated to deal with the datasets as the default option. In addition, the tool can also unify the missing points for each model-reference pair separately by modifying the argument ComMask_On. Further, whether to unify missing points across all variables of one model can also be chosen with the help of the argument Unify_VarMiss.

The script can calculate the statistics either for a single variable or for multiple variables (Fig. 4). The left dotted blue box in Fig. 4 shows the calculation process for single variable evaluation (SVE). The centered and uncentered modes calculate the centered and uncentered statistics, respectively.
Note that the calculated statistical metrics rely on the type of input variable. If it is a scalar field, uCORR (CORR), rms (SD), and RMSD (cRMSD) are calculated in the uncentered (centered) mode. With regard to a vector field, VSC (cVSC), RMSL (cRMSL), and RMSVD (cRMSVD) are calculated in the uncentered (centered) mode. In addition, two skill scores defined by Taylor (2001), $S_{1}$ and $S_{2}$, are computed in both the centered and uncentered modes for a scalar variable. Similarly, $S_{v 1}$ and $S_{v 2}$ are calculated for a vector variable (Xu et al., 2016). After the calculation, these statistics are saved in a file that can be used to generate a Taylor diagram or VFE diagram.

In terms of the MVIE (dotted green box in Fig. 4), the statistics of individual variables (i.e., scalar variables and vector variables) are calculated first. After the evaluation for each individual variable, all variables are normalized by the respective rms values of the reference and are grouped into 
a multivariable integrated field for the calculation of multivariable statistics. To consider the relative importance of various variables, weights can be added to each variable after normalization through the argument Wgt_var (Table 1). In the centered mode, either VME or MEVM is computed for a vector variable and the multivariable field based on the argument Cal_VME (Table 1). When MEVM is chosen, if the individual 2-D vector variable exists, the MEVD is also calculated for it. Finally, both the centered and uncentered MISSs are calculated. If more than one observational dataset is available, the statistics between each observation and the reference are calculated to take the observational uncertainty into account.

After the calculation, the statistics calculated above are written to a new NetCDF file specified by the MVIE_filename argument. Meanwhile, the ranges of these statistics can be printed on the screen if the Print_stats_r is set to True, helping users to set the color levels in the metrics table (Figs. 5, 6).

Similarly, users can modify parameters in Plot_MVIE.ncl to control the display of a figure or table. Users can also modify the attribute parameters for the VFE diagram and the metrics table. A detailed explanation and default values can be found in the Plot_MVIE.ncl script. Users can choose to create the VFE diagram, the metrics table, or both. Interpretations of plots are discussed in detail with examples in Sect. 5. In addition, all arguments in the MVIETool and their descriptions are summarized in readme.namelist for users' reference. More detailed explanations of the arguments can be found in the user guide of MVIETool.

\section{Application of the tool}

To illustrate the application of the tool, monthly mean datasets of 10 CMIP5 models (Table A2 in the Appendix) derived from the first ensemble run of historical experiments during the period from 1961 to 2000 are used. The variables used include climatological mean $600 \mathrm{hPa}$ specific humidity (Q600), SLP, SST, $850 \mathrm{hPa}$ temperature (T850), $850 \mathrm{hPa}$ $2-\mathrm{D}$ vector wind (uv850), and $200 \mathrm{hPa} 2-\mathrm{D}$ vector wind (uv200) in spring (March-April-May), summer (June-JulyAugust), autumn (September-October-November), and winter (December-January-February). We assessed the model's ability to simulate these variables in the Northern Hemisphere. The mean of two sets of reanalysis data is used as a reference: the Japan Meteorological Agency and the Central Research Institute of Electric Power Industry Reanalysis-55 (JRA55) and the National Centers for Environmental Prediction/National Center for Atmospheric Research Reanalysis Project (NNRP). All datasets are regridded to a common resolution of $2.5^{\circ} \times 2.5^{\circ}$ using a bilinear interpolation method before the evaluation. A common mask of missing value is used for all model and reanalysis datasets in each season.

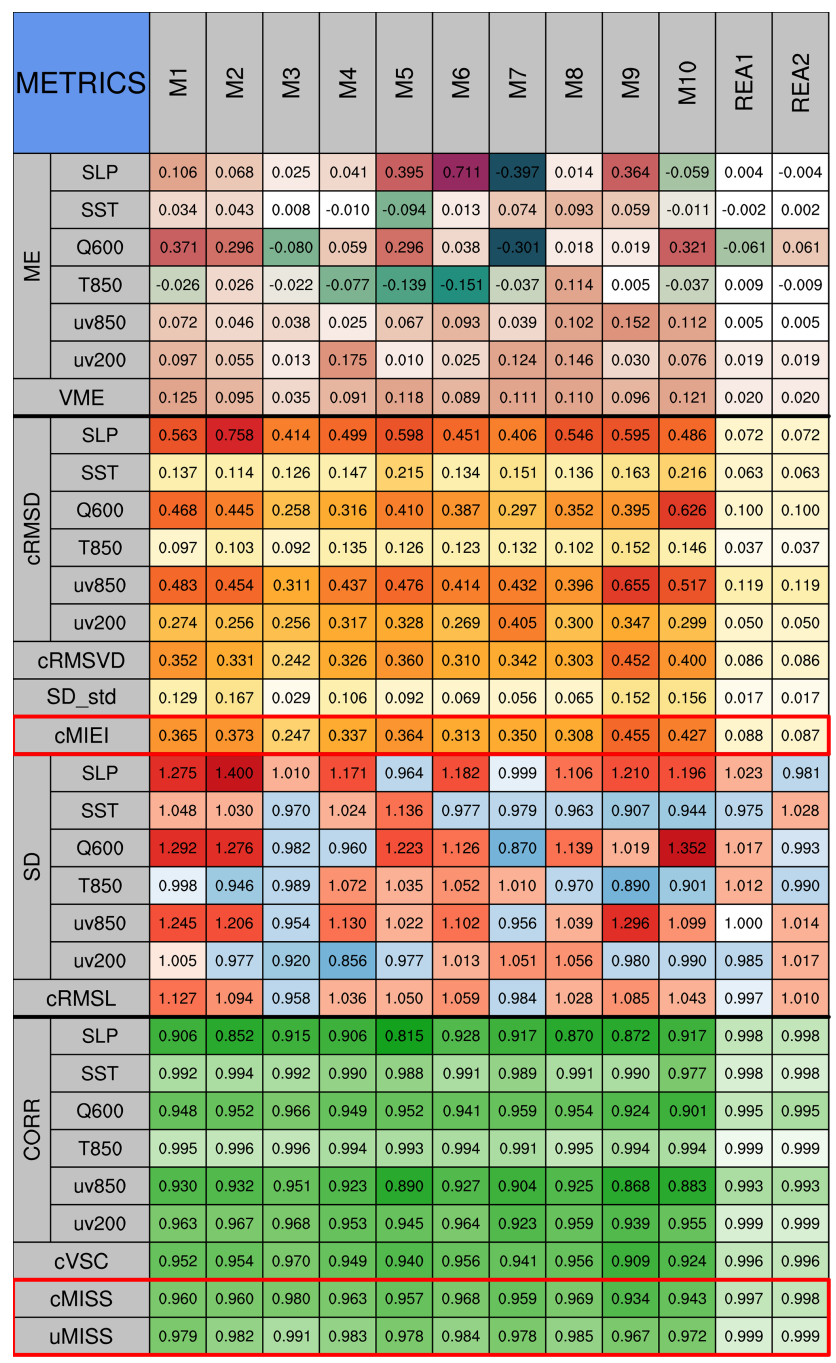

Figure 5. Metrics table of centered statistics. The table evaluates the performance of 10 CMIP5 models in simulating climatological mean (1961-2000) 600 hPa specific humidity (Q600), sea level pressure (SLP), sea surface temperature (SST), $850 \mathrm{hPa}$ temperature (T850), $850 \mathrm{hPa}$ winds (uv850) and $200 \mathrm{hPa}$ winds (uv200) in the Northern Hemisphere in autumn (September-October-November). $\mathrm{ME}$ (VME) is the error of the mean scalar or vector (multivariable) fields. cRMSD (cRMSVD) is the overall difference in scalar or vector (multivariable) anomaly fields between model and observation. CORR (cVSC) and SD (cRMSL) are the pattern similarity and amplitude of the anomaly fields for the individual variable (multivariable field). SD_std is the standard deviation of SD values. cMISS (uMISS) is the multivariable integrated skill score of the anomaly (original) fields, which is calculated with SD (rms) values and cVSC (VSC). cMIEI is the centered multivariable integrated index. The factor $F$ in cMISS and uMISS is 2. The colors represent the values of statistical metrics. The lighter colors indicate better model performance. 


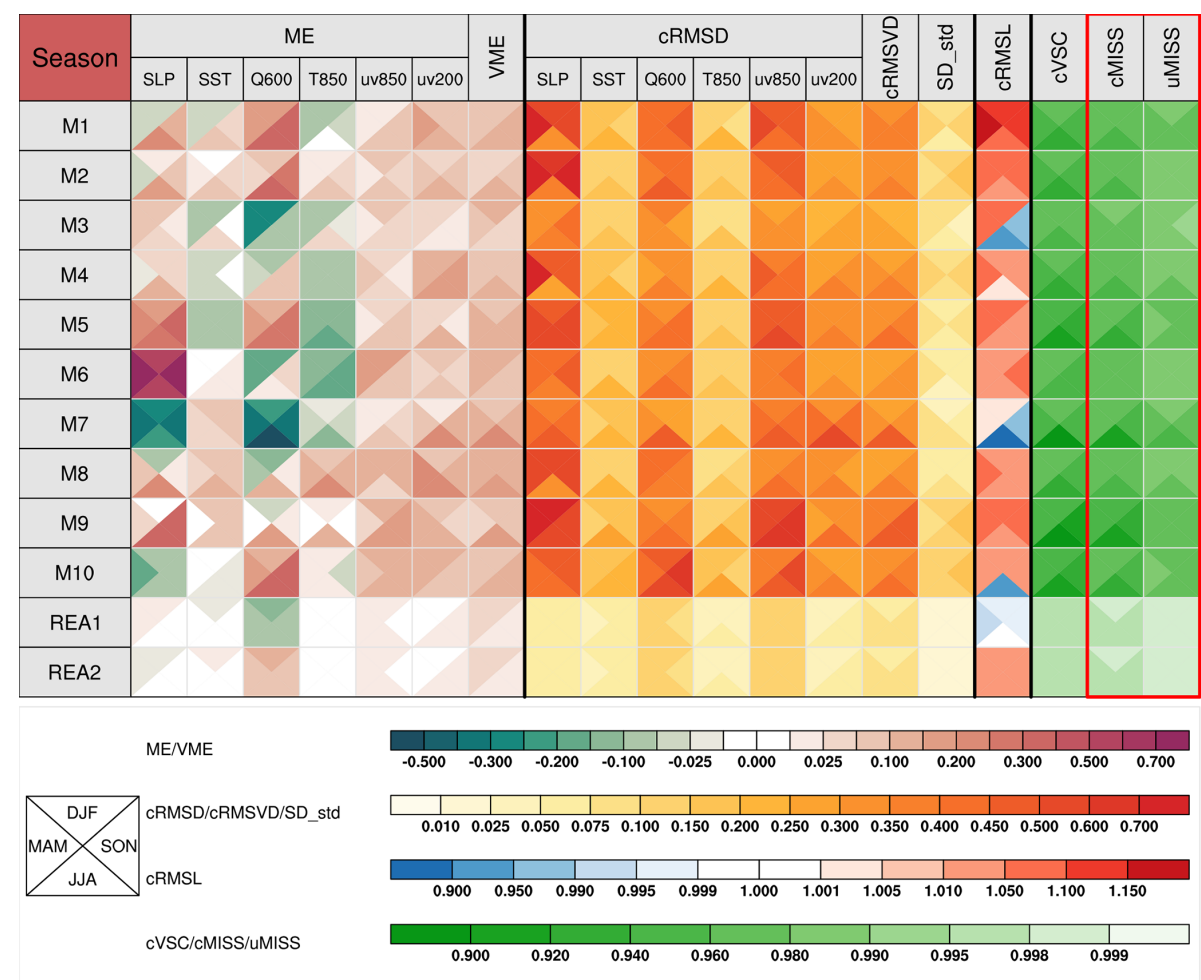

Figure 6. Metrics table of centered statistics. These models are used to simulate climatological means of 600 hPa specific humidity (Q600), SLP, SST, $850 \mathrm{hPa}$ temperature (T850), $850 \mathrm{hPa}$ winds (uv850) and $200 \mathrm{hPa}$ winds (uv200), of the Northern Hemisphere in four seasons, spring (March-April-May), summer (June-July-August), autumn (September-October-November), and winter (December-JanuaryFebruary). Each square is divided into four triangles, representing model performance in different seasons, as shown in the bottom-left legend. The colored bars for different statistical metrics are shown below the table.

\subsection{Metrics table}

The metrics table can show various model performance metrics in terms of individual variables and multivariable integrated field, as well as the overall model skill scores in either centered or uncentered mode. Figure 5 shows the metrics table of various statistical metrics, which evaluates six climatological mean fields - SLP, SST, Q600, T850, uv850, and uv200 - with centered statistical metrics. The filled color of each grid cell represents the value of statistical metric. Lighter colors indicate the model statistics are closer to observation, and vice versa. The corresponding color bars can be shown below the metric table such as those in Fig. 6 . Different types of statistics are separated from each other by a thick black line. To facilitate the comparison of the metrics from different variables, in the centered mode, the SD (cRMSL), cRMSD (cRMSVD), and ME (VME) of the models are normalized by dividing by the corresponding SD (cRMSL) of the reference. In the uncentered mode, rms (RMSL) and RMSD (RMSVD) are normalized using rms (RMSL).

The metrics table of the centered statistics decomposes the original field into mean and anomaly fields for evaluation. The anomaly fields are further evaluated from the per- spective of pattern similarity, variance, and overall difference between the model and observation. The metrics table can clearly explain how much of the overall error comes from the mean error (ME, VME), the amplitude error of the anomaly field (SD, cRMSL), or the error in pattern similarity of the anomaly field (CORR, cVSC). For example, the ME and cRMSD of M1 in simulating SLP are 0.106 and 0.563 , respectively, indicating that the overall error is caused mainly by the error in the anomaly field (Fig. 5). The cRMSD can be further attributed to the poor amplitude (1.275) and pattern similarity (0.906), which can be shown more clearly in a Taylor diagram or VFE diagram. Similarly, one can also decompose model errors into mean error (VME) and overall error of the anomaly field (cRMSVD) in terms of the simulation of multiple variables.

To summarize and rank the overall performance of a model in simulating multiple fields, the MISSs in both centered and uncentered modes are provided in the metrics table and are expected to provide a more accurate evaluation compared with MIEI. Figure 5 shows that M2 ranks eighth out of 10 models when referring to the values of the centered MIEI (cMIEI), while it ranks fifth based on the cMISS. The main reason is that cMIEI is sensitive to the error in SDs, particularly for an SD greater than 1. For example, the SD of 
SLP in M2 is 1.4 and it contributes about 0.027 to the first term on the right-hand side of Eq. (7a). The cVSC of M2 is 0.954 , which contributes about 0.092 to the second term on the right-hand side of Eq. (7a). Referring to the definition of $L_{A_{m}}^{*}$ (Eq. 8), 1.4 is equivalent to its reciprocal, 0.714, in the sense of model performance. However, the SD of 0.714 contributes only about 0.014 to the first term on the righthand side of Eq. (9). Thus, the MISS is equally sensitive to the model's abilities to simulate pattern similarity and amplitude. In addition, cMISS (uMISS) also allows us to adjust the relative importance of SD (rms) values and cVSC (VSC) based on the application (Eq. 10).

The MVIETool can be used in a very flexible way by modifying the arguments in the main scripts. A comparison of Fig. 5 with Fig. 6 helps to explain the flexibility. For example, users can choose the statistics to be displayed. In Fig. 6, only a few statistical metrics are displayed in comparison with Fig. 5. Unlike Fig. 5, Fig. 6 divides each grid cell into four triangles, representing model performance in each of the four seasons. Currently, the grid cell can be divided into two or four triangles. If no value of metrics is displayed in the grid cell, colored bars and a box legend are provided for reference. Moreover, considering that the relative number of models compared to the variables and statistical metrics may vary with the application, the MVIETool allows users to transpose the metrics table into a portrait or landscape orientation. For example, the model labels (statistical metrics and variables) can be arranged on the top or the left of the metrics table. A more detailed technical introduction for plotting is provided in the user guide.

\subsection{VFE diagram}

The VFE diagram is used to measure the model's ability to simulate the original (anomaly) vector or multiple fields in terms of three statistics: RMSL (cRMSL), VSC (cVSC), and RMSVD (cRMSVD). Figure 7 is the VFE diagram for a vector variable generated by the MVIETool. It assesses the climatological mean $850 \mathrm{hPa}$ vector winds of the Northern Hemisphere in autumn derived from 10 CMIP5 models (M1M10) during the period 1961-2000. Since the anomaly field of $850 \mathrm{hPa}$ vector wind is considered, cRMSL, cVSC, and cRMSVD are shown in the diagram. The construction of the VFE diagram is based on the geometric relationship (Eq. 15) between the three statistics. Thus, in this diagram, the azimuthal position gives VSC (cVSC), the radial distance from the origin indicates RMSL (cRMSL), and the distance between the model and the reference points provides RMSVD (cRMSVD). Similar to the metrics table, RMSL (cRMSL) and RMSVD (cRMSVD) were normalized by the RMSL (cRMSL) of the reference to facilitate an intercomparison between different variables. The VFE diagram can clearly show how much of the overall difference between model and observation is caused by poor pattern similarity and how much is due to the difference in the field amplitude (Xu et al., 2017).

Besides, a horizontal red line is shown in Fig. 7 centered at the "REF" point on $x$ axis, the length of which can represent the observational uncertainty. Here, we use the areaweighted mean of standard deviations $\left(\mathrm{M}_{\mathrm{SD}}\right)$ derived from multiple observations as the estimation of the observational uncertainty:

$\mathrm{M}_{\mathrm{SD}}=\frac{\sum_{i=1}^{M} \sum_{j=1}^{N} w_{j} \cdot \mathrm{SD}_{i j}^{*}}{M \cdot N}, \mathrm{SD}_{i j}^{*}=\frac{\mathrm{SD}_{i j}^{\mathrm{obs}}}{\mathrm{SD}_{i j}^{t}}$,

where $j(i)$ represents the grid (variable) index and $w_{j}$ is the area weighting. $\mathrm{SD}_{i j}^{\mathrm{obs}}$ is the standard deviation of multiple observations, which is calculated using the climatologies of REA1 and REA2 (Fig. 7). Clearly, more observational data are desirable to derive a statistically meaningful standard deviation. Here, we only aim to illustrate how to show observational uncertainty in the VFE diagram. $\mathrm{SD}_{i j}^{t}$ represents the interannual standard deviation of the reference, which is derived from the 40-year time series in autumn from 1961 to 2000. $\mathrm{M}_{\mathrm{SD}}$ is illustrated with the red line in Fig. 7 and it summarizes the mean dispersion of multiple observations in all grids for $M$ variables, which can roughly represent the overall uncertainty of observations.

Figure 7a is the same as Fig. 7b except that Fig. 7a applies area weighting to the statistics, but Fig. $7 \mathrm{~b}$ does not. Note that for some models (e.g., M2, M4, and M5), there is a relatively large difference between the statistics without and with area weighting, including $\mathrm{M}_{\mathrm{SD}}$. We recommend taking area weighting into account in model evaluation of a spatial field, especially for regions covering a broad span of latitudes. Furthermore, the tool can also generate a VFE diagram with SD (rms), CORR (uCORR), and cRMSD (RMSD), and in this situation it is the same as the Taylor diagram (Taylor, 2001; Xu et al., 2016).

\section{Summary}

In this paper, we have improved the MVIE method and developed the MVIETool to support the evaluation of model performance using this method. The improved MVIE method can evaluate overall model performance in simulating multiple scalar variables and vector variables. In addition, we consider area weighting in the definition of statistics, which is important for the evaluation of spatial fields on a longitude and latitude mesh grid. Based on MIEI, we further define a more accurate multivariable integrated skill score, termed MISS, to evaluate and rank the overall model performance in simulating multiple variables. Similar to MIEI, MISS also takes both amplitude and the pattern similarity into account, but it is a normalized index with the maximum value of 1 representing a perfect model. MISS is also flexible and able to adjust the relative importance between the pattern similarity and amplitude. 
(a)

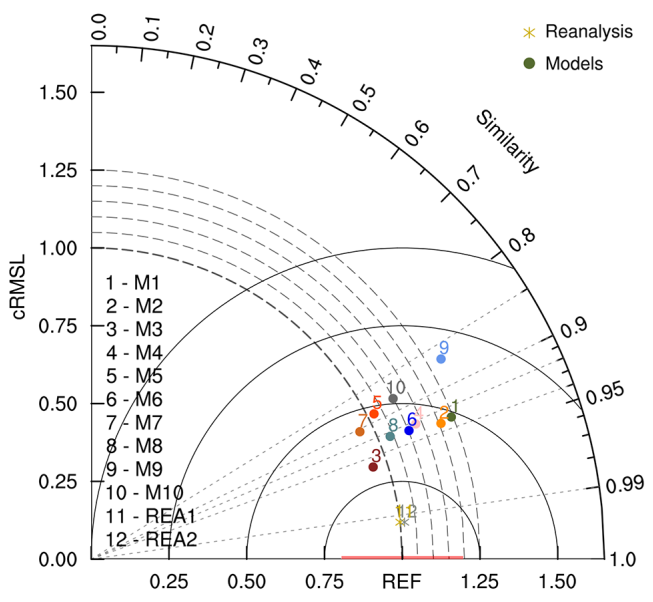

(b)

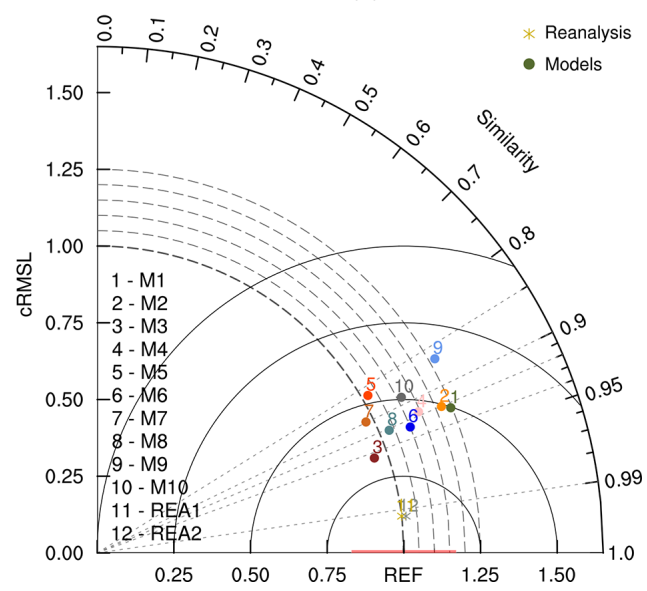

Figure 7. VFE diagram. This describes the climatological mean autumn (September-October-November) $850 \mathrm{hPa}$ wind fields derived from the different CMIP5 models (M1-M10) in the Northern Hemisphere. The azimuthal position gives cVSC, the radial distance from the origin indicates cRMSL, and the distance between the model and the reference points provides cRMSVD. The cRMSL and the cRMSVD are normalized by the cRMSL derived from the mean of two reanalysis datasets (REA1, REA2). The area weighting is considered in the statistical metrics in panel (a) but not in (b). The observational uncertainty is indicated by the horizontal red line centered at the REF point. Here, we use $\mathrm{M}_{\mathrm{SD}}$ value derived from REA1 and REA2 to estimate observational uncertainty.

A Multivariable Integrated Evaluation Tool, MVIETool (version 1.0), was developed in NCL code to facilitate the evaluation and intercomparison of model performance. The tool provides two modes of statistics, the uncentered mode and the centered mode, for different requirements of evaluation. The uncentered statistics, such as RMSL, VSC, and RMSVD, evaluate the model performance in terms of the original field. In contrast, the centered statistics evaluate model performance in simulating anomaly fields. In practice, some variables are dependent on each other to a certain extent, such as the 850 and $700 \mathrm{hPa}$ temperatures, and thus contain redundant information. To adjust the relative importance of various variables, we also take into consideration variable weighting in the statistics of the multivariable fields.

The MVIETool primarily consists of two main scripts with one for statistical metrics calculation and the other one for plotting. The tool is programmed to handle NetCDF data as input with a fixed format. Users can control the evaluation by setting arguments at the beginning of the main script. The statistics are shown in the VFE diagram and/or the metrics table, which provide a valuable visual overall evaluation of model performance. We demonstrated the utility of the MVIETool through three examples of 10 CMIP5 models in Sect. 5. The improved MVIE method, together with the MVIETool, is expected to assist researchers to efficiently evaluate model performance in terms of multiple fields.

To make the evaluation methods available to more users, we also develop the MVIETool with Python3. Currently, MVIETool 1.0 only provides some basic function to calculate statistics and generate figures for MVIE. We will continue to develop the tool to support more comprehensive evaluation.
For example, the area weighting is only valid for the regular grid in MVIETool 1.0. In terms of irregular grids, the area weighting can be derived from an additional data file that contains the grid area of each grid. To address observation uncertainty, the tool compares each individual observation against the average of multiple observations, and the spread across various observations is taken as a measure of observational uncertainty. Another approach is to calculate the standard deviation of multiple observations as uncertainty estimation at present, which is also very basic. It warrants further investigation to develop a more sophisticated method that can estimate the impacts of observational uncertainty on model evaluation. In addition, no significance test is available yet for difference between two vector fields as well as the multivariable statistics, which also warrants for development in the future.

Furthermore, the Earth System Model Evaluation Tool (ESMValTool; Eyring et al., 2016; Weigel et al., 2020) is a systematic and efficient tool for model evaluation, which has been widely used in related studies (e.g., Valdes et al., 2017; Righi et al., 2020; Waliser et al., 2020). It has many distinct advantages, such as providing the well-documented analysis and no need for preprocessing of evaluated datasets, compared with our tool. In the follow-up work, we would not only devote our efforts to making advances in the functionality of MVIETool but also intend to collaborate with the ESMValTool to include our package into it. In this way, users can benefit from the MVIETool with more convenience. 


\section{Appendix A}

Table A1. Table of statistics in the improved multivariable integrated method with their acronyms and descriptions.

\begin{tabular}{ll}
\hline Acronyms & Description \\
\hline Statistics for individual variables \\
\hline SD & Standard deviation \\
CORR & Correlation coefficient \\
cRMSD & Centered root-mean-square difference \\
rms & Root mean square \\
uCORR & Uncentered correlation coefficient \\
RMSD & Root-mean-square difference \\
ME & Mean error \\
\hline Statistics for multivariable integrated field \\
\hline cRMSL & Centered root-mean-square length (Eq. 11) \\
cVSC & Centered vector similarity coefficient (Eq. 12) \\
cRMSVD & Centered root-mean-square vector difference (Eq. 13) \\
SD_std & Standard deviation of SD values (Eq. 18) \\
RMSL & Root-mean-square length (Eq. 1) \\
VSC & Vector similarity coefficient (Eq. 2) \\
RMSVD & Root-mean-square vector difference (Eq. 3) \\
rms_std & Standard deviation of rms values (Eq. 6) \\
VME & Vector mean error (Eq. 14) \\
MEVM & Mean error of vector magnitude \\
MEVD & Mean error of vector direction \\
\hline Index for summarizing overall performance \\
\hline MIEI & Multivariable integrated evaluation index (Eq. 7a) \\
cMIEI & Centered multivariable integrated evaluation index \\
MISS & Multivariable integrated skill score \\
cMISS & Centered multivariable integrated skill score \\
uMISS & Uncentered multivariable integrated skill score (Eqs. 8-10) \\
\hline Observational uncertainty \\
\hline MSD & Mean of standard deviation derived from multiple observations (Eq. 20) \\
\hline & \\
\hline
\end{tabular}


Table A2. Model names, institution, and horizontal resolution for 10 CMIP5 models (M1-M10) used in the paper.

\begin{tabular}{|c|c|c|c|}
\hline & Model & Institution & Horizontal resolution \\
\hline M1 & BNU-ESM & $\begin{array}{l}\text { College of Global Change and Earth System Science, Beijing } \\
\text { Normal University (China) }\end{array}$ & $2.81^{\circ} \times 2.81^{\circ}$ \\
\hline M2 & CCSM4 & $\begin{array}{l}\text { NCAR (National Center for Atmospheric Research), Boulder } \\
\text { (USA) }\end{array}$ & $1.25^{\circ} \times 0.94^{\circ}$ \\
\hline M3 & CNRM-CM5 & $\begin{array}{l}\text { Centre National de Recherches Meteorologiques/Centre Eu- } \\
\text { ropeen de Recherche et Formation Avancees en Calcul Scien- } \\
\text { tifique (France) }\end{array}$ & $1.41^{\circ} \times 1.41^{\circ}$ \\
\hline M4 & BCC-CSM1-1 & $\begin{array}{l}\text { Beijing Climate Center, China Meteorological Administration } \\
\text { (China) }\end{array}$ & $2.81^{\circ} \times 2.81^{\circ}$ \\
\hline M5 & FGOALS-g2 & $\begin{array}{l}\text { LASG, Institute of Atmospheric Physics, Chinese Academy of } \\
\text { Sciences; and CESS, Tsinghua University (China) }\end{array}$ & $2.81^{\circ} \times 3.05^{\circ}$ \\
\hline M6 & GFDL-ESM2M & Geophysical Fluid Dynamics Laboratory (USA) & $2.5^{\circ} \times 2.0^{\circ}$ \\
\hline M7 & GISS-E2-H & NASA Goddard Institute for Space Studies (USA) & $2.5^{\circ} \times 2.0^{\circ}$ \\
\hline M8 & MIROC4h & $\begin{array}{l}\text { Atmosphere and Ocean Research Institute (The University of } \\
\text { Tokyo), National Institute for Environmental Studies, and Japan } \\
\text { Agency for Marine-Earth Science and Technology (Japan) }\end{array}$ & $0.56^{\circ} \times 0.56^{\circ}$ \\
\hline M9 & MIROC-ESM-CHEM & $\begin{array}{l}\text { Atmosphere and Ocean Research Institute (The University of } \\
\text { Tokyo), National Institute for Environmental Studies, and Japan } \\
\text { Agency for Marine-Earth Science and Technology (Japan) }\end{array}$ & $2.81^{\circ} \times 2.79^{\circ}$ \\
\hline M10 & inmcm4 & Institute for Numerical Mathematics (Russia) & $2.0^{\circ} \times 1.5^{\circ}$ \\
\hline
\end{tabular}


Code and data availability. MVIETool 1.0 (coded with NCL or Python3) is written in open-source scripts and is uploaded as a supplement as a frozen version of MVIETool 1.0. The codes and relevant data to generate figures in this paper are also provided in the Supplement. Additionally, the MVIETool 1.0 code and relevant data are also available on the GitHub repository: https:/github.com/ Mengzhuo-Zhang/MVIETool (last access: 24 May 2021, Zhang, 2021). Here, we will update the codes with minor improvements and to fix bugs in future.

Supplement. The supplement related to this article is available online at: https://doi.org/10.5194/gmd-14-3079-2021-supplement.

Author contributions. $\mathrm{MZ}$ and $\mathrm{ZX}$ devised the evaluation method and wrote the paper. MZ coded MVIETool 1.0. All the authors discussed the results and commented on the paper.

Competing interests. The authors declare that they have no conflict of interest.

Acknowledgements. We thank the climate modeling groups involved in the CMIP project for producing and making available their model outputs. NCEP Reanalysis derived data were provided by the NOAA/OAR/ESRL PSL, Boulder, Colorado, USA, from their website at https://psl.noaa.gov/data/gridded/data.ncep. reanalysis.derived.html (last access: 20 May 2021). The JRA55 reanalysis data were provided from Japanese 55-year reanalysis projects carried out by the Japan Meteorological Agency (JMA).

Financial support. This research has been supported by the National Key Research and Development Program of China (grant nos. 2018YFA0606004 and 2017YFA0603803) and the National Science Foundation of China (grant nos. 41675105, 41775075, and 42075152).

Review statement. This paper was edited by Andrea Stenke and reviewed by two anonymous referees.

\section{References}

Boer, G. and Lambert, S.: Second-order space-time climate difference statistics, Clim. Dyn., 17, 213-218, https://doi.org/10.1007/PL00013735, 2001.

Eyring, V., Bony, S., Meehl, G. A., Senior, C. A., Stevens, B., Stouffer, R. J., and Taylor, K. E.: Overview of the Coupled Model Intercomparison Project Phase 6 (CMIP6) experimental design and organization, Geosci. Model Dev., 9, 1937-1958, https://doi.org/10.5194/gmd-9-1937-2016, 2016.

Eyring, V., Cox, P.M., Flato, G. M., Gleckler, P. J., Abramowitz, G, Caldwell, P., Collins, W. D., Goer, B. K., Hall, A. D., Hoffman, F. M., Hurtt, G. C., Jahn, A., Jones, C. D., Klein, S. A.,
Krasting, J. P., Kwiatkowski, L., Lorenz, R., Maloney, E., Meehl, G. A., Pendergrass, A. G., Pincus, R., Ruane, A. C., Russell, J. L., Sanderson, B. M., Santer, B. D., Sherwood, S. C., Simpon, I. R., Stouffer, R. J., and Williamson M. S.: Taking climate model evaluation to the next level, Nat. Clim. Change, 9, 102110, https://doi.org/10.1038/s41558-018-0355-y, 2019.

Eyring, V., Righi, M., Lauer, A., Evaldsson, M., Wenzel, S., Jones, C., Anav, A., Andrews, O., Cionni, I., Davin, E. L., Deser, C., Ehbrecht, C., Friedlingstein, P., Gleckler, P., Gottschaldt, K.D., Hagemann, S., Juckes, M., Kindermann, S., Krasting, J., Kunert, D., Levine, R., Loew, A., Mäkelä, J., Martin, G., Mason, E., Phillips, A. S., Read, S., Rio, C., Roehrig, R., Senftleben, D., Sterl, A., van Ulft, L. H., Walton, J., Wang, S., and Williams, K. D.: ESMValTool (v1.0) - a community diagnostic and performance metrics tool for routine evaluation of Earth system models in CMIP, Geosci. Model Dev., 9, 1747-1802, https://doi.org/10.5194/gmd-9-1747-2016, 2016.

Flato, G., Marotzke, J., Abiodun, B., Braconnot, P., Chou, S. C., Collins, W., Cox, P., Driouech, F., Emori, S., Eyring, V., Forest, C., Gleckler, P., Guilyardi, E., Jakob, C., Kattsov, V., Reason, C., and Rummukainen, M.: Evaluation of Climate Models, in: Climate Change 2013: The Physical Science Basis. Contribution of Working Group I to the Fifth Assessment Report of the Intergovernmental Panel on Climate Change, edited by: Stocker, T. F., Qin, D., Plattner, G.K., Tignor, M., Allen, S. K., Boschung, J., Nauels, A., Xia, Y., Bex, V., and Midgleym, P. M., Cambridge University Press, Cambridge, UK, New York, NY, USA, 746748, 2013.

Gleckler, P. J., Taylor, K. E., and Doutriaux, C.: Performance metrics for climate models, J. Geophys. Res., 113, D06104, https://doi.org/10.1029/2007JD008972, 2008.

Huang, F., Xu, Z., and Guo, W.: Evaluating vector winds in the Asian-Australian monsoon region simulated by 37 CMIP5 models, Clim. Dyn., 53, 491-507, https://doi.org/10.1007/s00382018-4599-z, 2019.

Huang, F., Xu, Z., and Guo, W.: The linkage between CMIP5 climate models' abilities to simulate precipitation and vector winds. Clim. Dyn., 54, 4953-4970, https://doi.org/10.1007/s00382-02005259-6, 2020.

Knutti, R., Masson, D., and Gettelman, A.: Climate model genealogy: Generation CMIP5 and how we got there, Geophys. Res. Lett., 40, 1194-1199, https://doi.org/10.1002/grl.50256, 2013.

Kravitz, B., Robock, A., Boucher, O., Schmidt, H., Taylor, K. E., and Stenchikov, G.: The Geoengineering Model Intercomparison Project (GeoMIP), Atmos. Sci. Lett., 12, 162-167, https://doi.org/10.1002/asl.316, 2011.

Masson, D. and Knutti, R.: Spatial-Scale Dependence of Climate Model Performance in the CMIP3 Ensemble, J. Climate, 24, 2680-2692, https://doi.org/10.1175/2011JCLI3513.1, 2011.

Meehl, G. A., Moss, R., Taylor, K. E., Eyring, V., Stouffer, R. J., Bony, S., and Stevens, B.: Climate Model Intercomparisons: Preparing for the Next Phase, EOS T. Am. Geophys. Un., 95, 77-84, https://doi.org/10.1002/2014EO090001, 2014.

Pincus, R., Crispian, P. B., Hofmann, R. J. P., Taylor, K. E., and Glecker, P. J.: Evaluating the present-day simulation of clouds, precipitation, and radiation in climate models, J. Geophys. Res., 113, D14209, https://doi.org/10.1029/2007JD009334, 2008.

Pincus, R., Forster, P. M., and Stevens, B.: The Radiative Forcing Model Intercomparison Project (RFMIP): experimen- 
tal protocol for CMIP6, Geosci. Model Dev., 9, 3447-3460, https://doi.org/10.5194/gmd-9-3447-2016, 2016.

Righi, M., Hendricks, J., Lohmann, U., Beer, C. G., Hahn, V., Heinold, B., Heller, R., Krämer, M., Ponater, M., Rolf, C., Tegen, I., and Voigt, C.: Coupling aerosols to (cirrus) clouds in the global EMAC-MADE3 aerosol-climate model, Geosci. Model Dev., 13, 1635-1661, https://doi.org/10.5194/gmd-131635-2020, 2020.

Simpkins, G.: Process in climate modeling, Nat. Clim. Change., 7, 684-685, 2017.

Stouffer, R. J., Eyring, V., Meehl, G. A., Bony, S., Senior, C. Stevens, B., and Taylor, K. E.: CMIP5 scientific gaps and recommendations for CMIP6, B. Am. Meteorol. Soc., 98, 95-105, https://doi.org/10.1175/BAMS-D-15-00013.1, 2016.

Taylor, K. E.: Summarizing multiple aspects of model performance in a single diagram, J. Geophys. Res., 106, 7183-7192, 2001.

Valdes, P. J., Armstrong, E., Badger, M. P. S., Bradshaw, C. D., Bragg, F., Crucifix, M., Davies-Barnard, T., Day, J. J., Farnsworth, A., Gordon, C., Hopcroft, P. O., Kennedy, A. T., Lord, N. S., Lunt, D. J., Marzocchi, A., Parry, L. M., Pope, V., Roberts, W. H. G., Stone, E. J., Tourte, G. J. L., and Williams, J. H. T.: The BRIDGE HadCM3 family of climate models: HadCM3@Bristol v1.0, Geosci. Model Dev., 10, 3715-3743, https://doi.org/10.5194/gmd-10-3715-2017, 2017.

Waliser, D., Gleckler, P. J., Ferraro, R., Taylor, K. E., Ames, S., Biard, J., Bosilovich, M. G., Brown, O., Chepfer, H., Cinquini, L., Durack, P. J., Eyring, V., Mathieu, P.-P., Lee, T., Pinnock, S., Potter, G. L., Rixen, M., Saunders, R., Schulz, J., Thépaut, J.-N., and Tuma, M.: Observations for Model Intercomparison Project (Obs4MIPs): status for CMIP6, Geosci. Model Dev., 13, 29452958, https://doi.org/10.5194/gmd-13-2945-2020, 2020.

Watterson, I. G.: Non-Dimensional Measures Of Climate Model Performance, Int. J. Climatol., 16, 379-391, 1996.
Weigel, K., Bock, L., Gier, B. K., Lauer, A., Righi, M., Schlund, M., Adeniyi, K., Andela, B., Arnone, E., Berg, P., Caron, L.-P., Cionni, I., Corti, S., Drost, N., Hunter, A., Lledó, L., Mohr, C. W., Paçal, A., Pérez-Zanón, N., Predoi, V., Sandstad, M., Sillmann, J., Sterl, A., Vegas-Regidor, J., von Hardenberg, J., and Eyring, V.: Earth System Model Evaluation Tool (ESMValTool) v2.0 diagnostics for extreme events, regional and impact evaluation and analysis of Earth system models in CMIP, Geosci. Model Dev. Discuss. [preprint], https://doi.org/10.5194/gmd-2020-244, in review, 2020.

$\mathrm{Xu}, \mathrm{Z}$. and Han, Y.: Comments on "DISO: A rethink of Taylor diagram”, Int. J. Climatol., 40, 2506-2510, https://doi.org/10.1002/joc.6359, 2019.

$\mathrm{Xu}, \mathrm{Z}$., Han, Y., and Fu, C.: Multivariable integrated evaluation of model performance with the vector field evaluation diagram, Geosci. Model Dev., 10, 3805-3820, https://doi.org/10.5194/gmd-10-3805-2017, 2017.

$\mathrm{Xu}$, Z., Hou, Z., Han, Y., and Guo, W.: A diagram for evaluating multiple aspects of model performance in simulating vector fields, Geosci. Model Dev., 9, 4365-4380, https://doi.org/10.5194/gmd-9-4365-2016, 2016.

Zhang, M.-Z.: MVIETool, GitHub, available at: https://github.com/ Mengzhuo-Zhang/MVIETool, last access: 24 May, 2021.

Zhou, T., Turner, A. G., Kinter, J. L., Wang, B., Qian, Y., Chen, X., Wu, B., Wang, B., Liu, B., Zou, L., and He, B.: GMMIP (v1.0) contribution to CMIP6: Global Monsoons Model Inter-comparison Project, Geosci. Model Dev., 9, 3589-3604, https://doi.org/10.5194/gmd-9-3589-2016, 2016. 